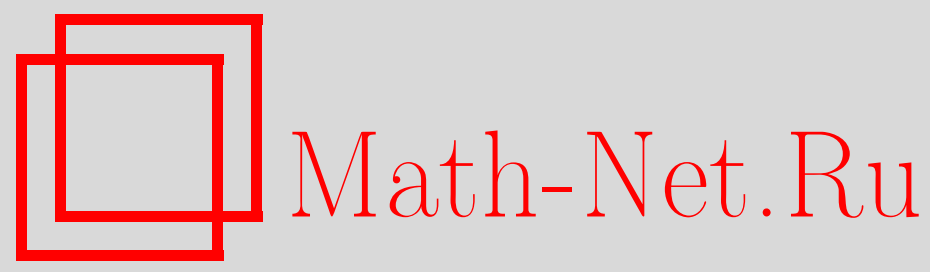

В. А. Юрко, О восстановлении несамосопряженных дифференциальных систем на полуоси по матрице Вейля, Матем. заметки, 2004, том 76, выпуск 2, 316-320

DOI: https://doi.org/10.4213/mzm572

Использование Общероссийского математического портала Math-Net.Ru подразумевает, что вы прочитали и согласны с пользовательским соглашением http://www . mathnet.ru/rus/agreement

Параметры загрузки:

IP: 54.196 .121 .252

26 апреля 2023 г., 12:52:35

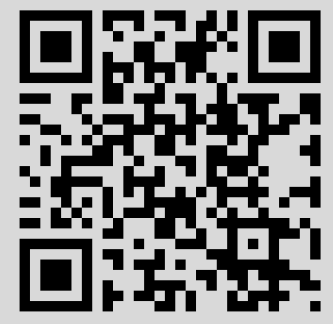




\section{О ВОССТАНОВЛЕНИИ НЕСАМОСОПРЯЖЕННЫХ ДИФФЕРЕНЦИАЛЬНЫХ СИСТЕМ НА ПОЛУОСИ ПО МАТРИЦЕ ВЕЙЛЯ}

\section{В. А. Юрко}

1. В статье исследуется обратная спектральная задача для следующей системы дифференциальных уравнений на полуоси:

$$
\ell Y(x):=Q_{0} Y^{\prime}(x)+Q(x) Y(x)=\rho Y(x), \quad x>0 .
$$

Здесь $Y=\left[y_{k}\right]_{k=\overline{1, n}}^{T}$ - вектор-столбец ( $T$ - знак транспонирования), $\rho$ - спектральный параметр, $Q_{0}=\operatorname{diag}\left[q_{k}\right]_{k=\overline{1, n}}, Q(x)=\left[q_{k j}(x)\right]_{k, j=\overline{1, n}}$, где $q_{k} \neq 0, k=\overline{1, n}$ - комплексные числа, $q_{k k}(x) \equiv 0$, а функции $q_{k j}(x), k \neq j$, являются комплексно-значными, абсолютно непрерывными и $q_{k j}^{(\nu)}(x) \in L(0, \infty), \nu=0,1$. Матрица $Q(x)$ назьвается потенциалом.

Обратные задачи спектрального анализа состоят в восстановлении операторов по их спектральньм характеристикам. Такие задачи часто встречаются в математике, механике, физике, электронике, геофизике, метеорологии и других областях естествознания и техники. Обратные задачи для систем обыкновенных дифференциальных уравнений в различных постановках исследовались многими авторами. Большинство работ посвящено обратным задачам для систем Дирака, AKNS и их обобщениям на случай произвольного $n>2$ (см. [1]-[3] и литературу в них). Основньм инструментом исследования здесь является метод оператора преобразования, и полученные результаты в основном аналогичны результатам для оператора Штурма-Лиувилля [4]-[6]. Однако для систем (1) общего вида с произвольными комплексными $q_{k}$ и интегрируемой $Q(x)$ обратная задача оказьвается существенно более трудной для исследования. Операторы преобразования в общем случае имеют весьма сложную структуру, что затрудняет их применение при решении обратной задачи. Исключение составляет случай аналитического потенциала, когда операторы преобразования имеют такой же “треугольный” вид, как и для систем Дирака и AKNS. Этот частный случай изучался в [7]. Исследованию обратных задач для систем (1) общего вида с произвольными комплексными $q_{k}$ посвящено немного работ, причем в основном эти работы посвящены случаю оси. Отметим важные работы [8], [9], в которых с использованием задачи Римана для аналитических функций изучалась обратная задача рассеяния для системы (1) на оси. Обратная задача для системы (1) на полуоси и на конечном интервале в общем случае еще не исследовалась.

В данной статье исследуется обратная задача для системы (1) на полуоси при произвольных комплексных $q_{k}$ и при произвольном поведении спектра. В качестве основной спектральной характеристики вводится и изучается так назьваемая матрица Вейля, которая является аналогом классической функции Вейля для оператора Штурма-Лиувилля и матрицы Вейля, введенной в [10] для дифференциальных операторов высших порядков. Основным методом исследования является метод спектральных отображений [11], [12]. Развивая идеи этого метода применительно к системам вида (1) и используя выявленные аналитические и структурные свойства матрицы Вейля, мы доказьваем теорему единственности решения обратной задачи по матрице Вейля и получаем конструктивную процедуру ее решения.

Обозначим $\beta_{k}=1 / q_{k}$. Пусть для определенности $\beta_{k} \neq \beta_{j}$ при $k \neq j$. Известно, что $\rho$-плоскость можно разбить на сектора

$$
S_{j}=\left\{\rho: \arg \rho \in\left(\theta_{j}, \theta_{j+1}\right)\right\}, \quad j=\overline{0,2 r-1}, \quad 0 \leqslant \theta_{0}<\theta_{1}<\cdots<\theta_{2 r-1}<2 \pi,
$$

в каждом из которых существует перестановка $i_{k}=i_{k}\left(S_{j}\right)$ чисел $1, \ldots, n$ такая, что для чисел $R_{k}=R_{k}\left(S_{j}\right)$ вида $R_{k}=\beta_{i_{k}}$ имеем

$$
\operatorname{Re}\left(\rho R_{1}\right)<\cdots<\operatorname{Re}\left(\rho R_{n}\right), \quad \rho \in S_{j} .
$$

Работа выполнена при поддержкегранта Министерства образования, грант № Е02-1.0-186, гранта “Университеты России”, грант № УР.04.01.042, и РФФИ, грант № 04-01-00007. 
Отметим, что число $2 r$ секторов $S_{j}$ зависит от расположения чисел $\left\{\beta_{k}\right\}_{k=\overline{1, n}}$ на комплексной плоскости, причем $1 \leqslant r \leqslant n(n-1) / 2$. Пусть дана матрица $h=\left[h_{\xi \nu}\right]_{\xi, \nu=\overline{1, n}}, \operatorname{det} h \neq 0$, где $h_{\xi \nu}-$ комплексные числа. Введем линейные формы $U(Y)=\left[U_{\xi}(Y)\right]_{\xi=\overline{1, n}}^{T}$ по формуле $U(Y)=h Y(0)$,

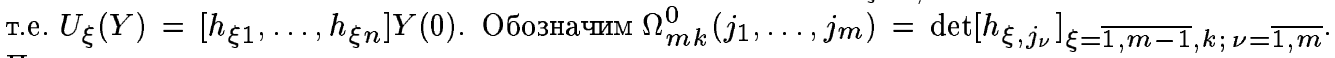
Предположим, что

$$
\Omega_{m m}^{0}\left(i_{1}, \ldots, i_{m}\right) \neq 0, \quad m=\overline{1, n-1},
$$

где $i_{k}=i_{k}\left(S_{j}\right)$ - вышеупомянутая перестановка для сектора $S_{j}, j=\overline{0,2 r-1}$. Условие (3) называется условием информативности для пары $L=(\ell, U)$. Системы, которые не удовлетворяют условию информативности, обладают качественно другими закономерностями при постановке и исследовании обратных задач и в данной работе не рассматриваются. Без ограничения общности считаем, что вьполняются следующие условия нормировки: $\operatorname{det} h=1$ и для некоторого фиксированного сектора (для определенности для сектора $S_{0}$ ) имеем $\Omega_{m m}^{0}\left(i_{1}, \ldots, i_{m}\right)=1, m=\overline{1, n-1}$. Везде в дальнейшем будем считать, что условия информативности и нормировки выполнены.

Пусть вектор-функции

$$
\Phi_{m}(x, \rho)=\left[\Phi_{k m}(x, \rho)\right]_{k=1, n}^{T}, \quad m=\overline{1, n},
$$

являются решениями системы (1) при условиях $U_{\xi}\left(\Phi_{m}\right)=\delta_{\xi m}, \xi=\overline{1, m}$, а также $\Phi_{m}(x, \rho)=$ $O\left(\exp \left(\rho R_{m} x\right)\right), x \rightarrow \infty, \rho \in S_{j}$ в каждом секторе $S_{j}$ со свойством (2). Эти условия однозначно определяют решения $\Phi_{m}(x, \rho)$. Положим $M_{m \xi}(\rho)=U_{\xi}\left(\Phi_{m}\right), \xi>m, M(\rho)=\left[M_{m \xi}(\rho)\right]_{m, \xi=\overline{1, n}}$, $M_{m \xi}(\rho)=\delta_{\xi m}$ при $\xi \leqslant m, \Phi(x, \rho)=\left[\Phi_{1}(x, \rho), \ldots, \Phi_{n}(x, \rho)\right]=\left[\Phi_{k m}(x, \rho)\right]_{k, m=\overline{1, n}}$. Функции $\Phi_{m}(x, \rho)$ и $M_{m \xi}(\rho)$ называются решениями Вейля и функииями Вейля соответственно. Матрица $M(\rho)$ называется матрицей Вейля или спектром $L=(\ell, U)$.

ПОСТАНОВКА ОБРАТнОЙ ЗАДАЧИ. Фиксируем $Q_{0}$, т.е. числа $\beta_{k}=1 / q_{k}, k=\overline{1, n}$, известны и фиксированы. Обратная задача ставится следующим образом: по заданной матрице Вейля $M(\rho)$ построить пару $L=(\ell, U)$.

2. Обозначим

$$
\Gamma_{j}=\left\{\rho: \arg \rho=\theta_{j}\right\}, \quad j=\overline{0,2 r-1}, \quad \Gamma_{2 r}:=\Gamma_{0} .
$$

Разрежем $\rho$-плоскость вдоль лучей $\Gamma_{j}$ и обозначим через $\Gamma_{j}^{ \pm}=\left\{\rho: \arg \rho=\theta_{j} \pm 0\right\}$ берега разрезов. Положим $\bar{S}_{j}=S_{j} \cup \Gamma_{j}^{+} \cup \Gamma_{j+1}^{-}$и обозначим

$$
\Sigma=\mathbb{C} \backslash\left(\bigcup_{j=0}^{2 r-1} \Gamma_{j}\right)=\bigcup_{j=0}^{2 r-1} S_{j}
$$

- $\rho$-плоскость без разрезов вдоль лучей $\Gamma_{j}$, и $\bar{\Sigma}=\bigcup_{j=0}^{2 r-1} \bar{S}_{j}$ - замыкание $\Sigma$ (различаем берега разрезов).

Зафйисируем $j=\overline{0,2 r-1}$. При $\rho \in \Gamma_{j}$ строгие неравенства в (2) в некоторых местах превращаются в равенства. Пусть $m_{i}=m_{i}(j), p_{i}=p_{i}(j), i=\overline{1, s}$, таковы, что при $\rho \in \Gamma_{j}$ выполнено

$$
\operatorname{Re}\left(\rho R_{m_{i}-1}\right)<\operatorname{Re}\left(\rho R_{m_{i}}\right)=\cdots=\operatorname{Re}\left(\rho R_{m_{i}+p_{i}}\right)<\operatorname{Re}\left(\rho R_{m_{i}+p_{i}+1}\right), \quad i=\overline{1, s},
$$

где $R_{k}=R_{k}\left(S_{j}\right)$. Положим

$$
N_{j}:=\left\{m: m=\overline{m_{1}, m_{1}+p_{1}-1}, \ldots, \overline{m_{s}, m_{s}+p_{s}-1}\right\},
$$

$J_{m}:=\left\{j: m \in N_{j}\right\}, \gamma_{m}=\bigcup_{j \in J_{m}} \Gamma_{j}$, и пусть $\Sigma_{m}=\mathbb{C} \backslash \gamma_{m}-\rho$-плоскость без разрезов вдоль лучей из $\gamma_{m}$, a $\bar{\Sigma}_{m}$ - замькание $\Sigma_{m}$ (различаем берега разрезов). Ясно, что область $\Sigma_{m}=\bigcup_{\nu} S_{m \nu}$ состоит из секторов $S_{m}$, каждый из которых является объединением нескольких секторов $S_{j}$ с одним и тем же набором $\left\{R_{\xi}\right\}_{\xi=\overline{1, m}}$. При $\xi=\overline{0, n-2}$ построим функции

$$
B_{m k}^{\xi}(\rho), \quad m=\overline{1, n-\xi-1}, \quad k=\overline{m+\xi+1, n},
$$

по следуюшим рекуррентным формулам:

$$
B_{m k}^{0}(\rho)=M_{m k}(\rho), \quad B_{m k}^{\xi}(\rho)=B_{m k}^{\xi-1}(\rho)-B_{m, m+\xi}^{\xi-1}(\rho) B_{m+\xi, k}^{0}(\rho) .
$$


Теорема 1. Матрица Вейля $M(\rho)=\left[M_{m k}(\rho)\right]_{m, k=\overline{1, n}}$ имеет следующие свойства.

1) Имеет место равенство $M_{m k}(\rho)=\delta_{m k}$ при $k \leqslant m$.

2) Функиии Вейля $M_{m k}(\rho), k>m$, являются аналитическими в $\Sigma_{m}$ за исключением не более чем счетного ограниченного множества $\Lambda_{m}^{\prime}$ полюсов и непрерывны в $\bar{\Sigma}_{m}$ (m.е. они непрерывны в каждом секторе $\bar{S}_{m \nu}$ ) за исключением ограниченного множества $\Lambda_{m}$. точнее, при $j \in J_{m}, \rho \in \Gamma_{j} \backslash \Lambda_{m}$ существуют конечнье предель

$$
M_{m k}^{ \pm}(\rho)=\lim M_{m k}(z), \quad z \rightarrow \rho, \quad z \in \Sigma_{m}, \quad \pm\left(\arg z-\theta_{j}\right)>0 .
$$

На $\gamma_{m}$ функиии $M_{m k}(\rho)$ имеют разрез.

3) При $|\rho| \rightarrow \infty, \rho \in \bar{S}_{j}$ имеет место асимптотическое равенство

$$
M_{m k}(\rho)=\mu_{m k}^{0}\left(S_{j}\right)+O\left(\rho^{-1}\right)
$$

əде

$$
\mu_{m k}^{0}\left(S_{j}\right)=\frac{\Omega_{m k}^{0}\left(i_{1}, \ldots, i_{m}\right)}{\Omega_{m m}^{0}\left(i_{1}, \ldots, i_{m}\right)}, \quad i_{k}=i_{k}\left(S_{j}\right) .
$$

4) Функиии $B_{\nu k}^{m-\nu}(\rho)$ являются аналитическими на $\Gamma_{j} \backslash \Lambda_{m}^{\prime}$ при $j \notin J_{m}, 1 \leqslant \nu \leqslant m \leqslant$ $n-1, m+1 \leqslant k \leqslant n$.

Доказательства этой и последующих теорем см. в [13]. Обозначим

$$
\Lambda=\bigcup_{m} \Lambda_{m}, \quad h^{*}=\left[h_{k \xi}^{*}\right]_{k, \xi=\overline{1, n}}:=Q_{0} h^{-1}, \quad R_{m}^{*}:=-R_{n-m+1} .
$$

Рассмотрим дифференциальную систему и линейные формы $L^{*}=\left(\ell^{*}, U^{*}\right)$ :

$$
\ell^{*} Z(x):=-Z^{\prime}(x) Q_{0}+Z(x) Q(x)=\rho Z(x), \quad U^{*}(Z)=Z(0) h^{*},
$$

где $Z=\left[z_{k}\right]_{k=\overline{1, n}}-$ вектор-строка. Тогда $U^{*}(Z)=\left[U_{n}^{*}(Z), \ldots, U_{1}^{*}(Z)\right]$, где $U_{n-\xi+1}^{*}(Z)=$ $Z(0)\left[h_{k \xi}^{*}\right]_{k=\overline{1, n}}^{T}$. Пусть вектор-функции $\Phi_{m}^{*}(x, \rho)=\left[\Phi_{k m}^{*}(x, \rho)\right]_{k=\overline{1, n}}, m=\overline{1, n}$ являются решениями (6) при условиях $U_{\xi}^{*}\left(\Phi_{m}^{*}\right)=\delta_{\xi m}, \xi=\overline{1, m}$, а также $\Phi_{m}^{*}(x, \rho)=O\left(\exp \left(\rho R_{m}^{*} x\right)\right), x \rightarrow \infty$, $\rho \in S_{j}$ в каждом секторе $S_{j}$ со свойством (2). Положим

$$
\begin{gathered}
\Phi^{*}(x, \rho)=\left[\Phi_{n-m+1}^{*}(x, \rho)\right]_{m=\overline{1, n}}^{T}=\left[\Phi_{n-m+1, k}^{*}(x, \rho)\right]_{m, k=\overline{1, n}} \\
M_{m k}^{*}(\rho)=U_{k}\left(\Phi_{m}^{*}\right), \quad M^{*}(\rho)=\left[M_{n-\xi+1, n-k+1}^{*}(\rho)\right]_{k, \xi=\overline{1, n}} .
\end{gathered}
$$

Можно доказать, что $M^{*}(\rho)=M^{-1}(\rho)$.

3. Наряду с $L=(\ell, U)$ рассмотрим пару $\widetilde{L}=(\tilde{\ell}, \widetilde{U})$ того же вида, но с другими матрицами $\widetilde{Q}$, $\tilde{h}$ (напомним, что матрица $Q_{0}$ известна априори и фиксирована). Везде в дальнейшем считаем, что если некоторый символ $\alpha$ обозначает объект, относящийся к $L$, то $\tilde{\alpha}$ обозначает аналогичньй объект, относящийся к $\widetilde{L}$, и $\hat{\alpha}:=\alpha-\tilde{\alpha}$. Приведем теперь теорему единственности решения обратной задачи восстановления $L$ по матрице Вейля.

Tеорема 2. Если $M(\rho)=\widetilde{M}(\rho)$, то $L=\widetilde{L}$. Таким образом, задание матрицы Вейля $M(\rho)$ однозначно определяет потенциал $Q(x)$ и матричу $h$.

СлЕдствиЕ 1. Задание чисел $\mu_{m k}^{0}\left(S_{j}\right)$ вида (5) при всех $m, k, j$ однозначно определяет матричу линейных форм $h=\left[h_{\xi \nu}\right]_{\xi, \nu=\overline{1, n}}$.

ДокАЗАтЕльство. В самом деле, если $Q(x) \equiv 0$, то $M_{m k}(\rho) \equiv \mu_{m k}^{0}\left(S_{j}\right), \rho \in \overline{S_{j}}$, при всех $m$, $k, j$, и наше утверждение следует из теоремы 2 . 
4. Центральную роль при решении обратной задачи играет здесь так называемое основное уравнение обратной задачи, которое является линейным особым интегральным уравнением (см. (8)). Доказана однозначная разрешимость основного уравнения в соответствующем банаховом пространстве. Опираясь на решение основного уравнения, мы получаем конструктивную процедуру решения обратной задачи восстановления $L$ по заданной матрице Вейля.

Пусть пара $\widetilde{L}=(\tilde{\ell}, \widetilde{U})$ выбрана так, что

$$
\widehat{M}(\rho)=O\left(\rho^{-1}\right), \quad|\rho| \rightarrow \infty,
$$

т.е. $\mu_{m k}^{0}\left(S_{j}\right)=\tilde{\mu}_{m k}^{0}\left(S_{j}\right)$ при всех $m, k, j$. Согласно (4) и следствию 1 условие (7) равносилно условию $h=\tilde{h}$. Например, можно взять $\widetilde{Q}(x) \equiv 0$.

В $\rho$-плоскости рассмотрим контур $\omega=\omega^{0} \cup \omega^{+} \cup \omega^{-}$(с обходом против часовой стрелки), где $\omega^{0}$ - ограниченный замкнутый контур, охватывающий множество $\Lambda \cup \widetilde{\Lambda} \cup\{0\}$ (т.е. $\Lambda \cup \widetilde{\Lambda} \cup\{0\} \subset$ $\left.\operatorname{int} \omega^{0}\right)$, и

$$
\omega^{ \pm}=\bigcup_{j=0}^{2 r-1} \omega_{j}^{ \pm}, \quad \omega_{j}^{ \pm}=\Gamma_{j}^{ \pm} \backslash \operatorname{int} \omega^{0} .
$$

Определим матрицы $A(\rho)=\left[a_{m k}(\rho)\right]_{m, k=\overline{1, n}}$ и $\widetilde{A}(\rho)=\left[\tilde{a}_{m k}(\rho)\right]_{m, k=\overline{1, n}}$ по формулам

$$
A(\rho)=\mathscr{N}^{*}(\rho) \widehat{\mathcal{N}}(\rho), \quad \widetilde{A}(\rho)=\widetilde{\mathcal{N}}^{*}(\rho) \widehat{\mathcal{N}}(\rho), \quad \text { где } \mathcal{N}(\rho)=(M(\rho))^{T}, \quad \mathcal{N}^{*}(\rho)=\left(M^{*}(\rho)\right)^{T} .
$$

Ясно, что, $a_{m k}(\rho)=0, \tilde{a}_{m k}(\rho)=0$ при $m \leqslant k$, а при $m>k$ имеем

$a_{m k}(\rho)=\sum_{\xi=k+1}^{m} M_{n-m+1, n-\xi+1}^{*}(\rho) \widehat{M}_{k \xi}(\rho), \quad \tilde{a}_{m k}(\rho)=\sum_{\xi=k+1}^{m} \widetilde{M}_{n-m+1, n-\xi+1}^{*}(\rho) \widehat{M}_{k \xi}(\rho)$.

Фиксируем $j=\overline{0,2 r-1}$. Пусть

$$
N_{j}=\left\{\overline{m_{1}, m_{1}+p_{1}-1}, \ldots, \overline{m_{s}, m_{s}+p_{s}-1}\right\}, \quad m_{i}-1, m_{i}+p_{i} \notin N_{j}, \quad i=\overline{1, s} .
$$

Рассмотрим матрицы

$$
A^{(j)}(\rho)=\left[a_{k \xi}^{(j)}(\rho)\right]_{k, \xi=\overline{1, n}}, \quad \widetilde{A}^{(j)}(\rho)=\left[\tilde{a}_{k \xi}^{(j)}(\rho)\right]_{k, \xi=\overline{1, n}}, \quad \rho \in \Gamma_{j}^{ \pm},
$$

где $a_{k \xi}^{(j)}(\rho)=a_{k \xi}(\rho), \tilde{a}_{k \xi}^{(j)}(\rho)=\tilde{a}_{k \xi}(\rho)$ при $m_{i} \leqslant \xi<k \leqslant m_{i}+p_{i}, i=\overline{1, s}$, и $a_{k \xi}^{(j)}(\rho)=\tilde{a}_{k \xi}^{(j)}(\rho)=0$ в остальных случаях. При $\rho \in \omega$ введем матрицы $A_{0}(\rho)$ и $\widetilde{A}_{0}(\rho)$ по формулам

$$
A_{0}(\rho)=\left\{\begin{array}{ll}
A^{(j)}(\rho), & \rho \in \omega_{j}^{ \pm}, \\
A(\rho), & \rho \in \omega^{0},
\end{array} \quad \widetilde{A}_{0}(\rho)= \begin{cases}\widetilde{A}^{(j)}(\rho), & \rho \in \omega_{j}^{ \pm} \\
\widetilde{A}(\rho), & \rho \in \omega^{0}\end{cases}\right.
$$

В контуре $\omega$ удобно склеить берега разрезов. Поэтому в $\rho$-плоскости мы рассмотрим контур

$$
\omega^{*}:=\omega^{0} \cup \omega^{1}, \quad \text { где } \omega^{1}=\bigcup_{j=0}^{2 r-1} \omega_{j}^{1}, \quad \omega_{j}^{1}:=\left\{\rho: \rho \in \Gamma_{j} \backslash \omega^{0}\right\}
$$

(с ориентацией в сторону возрастания модуля $\rho$ ). При $\rho \in \omega^{*}$ определим матрицы $\varphi(x, \rho), \tilde{\varphi}(x, \rho)$, $\widetilde{G}(x, \rho), \widetilde{S}(\rho)$ и $\tilde{r}(x, \mu, \rho)$ по формулам

$$
\begin{aligned}
& \varphi(x, \rho)=\left\{\begin{array}{ll}
{\left[\Phi^{+}(x, \rho), \Phi^{-}(x, \rho)\right],} & \rho \in \omega^{1}, \\
\Phi(x, \rho), & \rho \in \omega^{0},
\end{array} \quad \tilde{\varphi}(x, \rho)= \begin{cases}{\left[\tilde{\Phi}^{+}(x, \rho), \widetilde{\Phi}^{-}(x, \rho)\right],} & \rho \in \omega^{1}, \\
\widetilde{\Phi}(x, \rho), & \rho \in \omega^{0},\end{cases} \right. \\
& \widetilde{G}(x, \rho)=\left[\begin{array}{c}
-\left(A_{0}(\rho) \widetilde{\Phi}^{*}(x, \rho)\right)^{+} Q_{0} \\
\left(A_{0}(\rho) \widetilde{\Phi}^{*}(x, \rho)\right)^{-} Q_{0}
\end{array}\right], \quad \rho \in \omega^{1}, \quad \widetilde{G}(x, \rho)=A_{0}(\rho) \widetilde{\Phi}^{*}(x, \rho) Q_{0}, \quad \rho \in \omega^{0}, \\
& \widetilde{S}(\rho)=\left[\begin{array}{cc}
E-\frac{1}{2} A_{0}^{+}(\rho) & \frac{1}{2}\left(A_{0}(\rho) \widetilde{\mathcal{N}}^{*}(\rho)^{+} \widetilde{\mathcal{N}}^{-}(\rho)\right. \\
\frac{1}{2}\left(A_{0}(\rho) \widetilde{\mathcal{N}}^{*}(\rho)\right)^{-\widetilde{N}^{+}(\rho)} & E-\frac{1}{2} A_{0}^{-}(\rho)
\end{array}\right], \quad \rho \in \omega^{1}, \quad f^{ \pm}:=f_{\mid \omega^{ \pm}}, \\
& \widetilde{S}(\rho)=E-\frac{1}{2} A_{0}(\rho), \quad \rho \in \omega^{0}, \quad \tilde{r}(x, \mu, \rho)=\frac{\widetilde{G}(x, \mu) \tilde{\varphi}(x, \rho)}{\mu-\rho}, \quad \rho, \mu \in \omega^{*} .
\end{aligned}
$$


Tеорема 3. Справедливо соотношение

$$
\tilde{\varphi}(x, \rho)=\varphi(x, \rho) \widetilde{S}(\rho)+\frac{1}{2 \pi i} \int_{\omega^{*}} \varphi(x, \mu) \tilde{r}(x, \mu, \rho) d \mu, \quad \rho \in \omega^{*} .
$$

При каждом фиксированном $x \geqslant 0$ соотношение (8) является линейным интегральньм уравнением относительо $\varphi(x, \rho)$. Соотношение $(8)$ назьвается основным уравнением обратной задачи. Исследуем разрешимость основного уравнения (8). Для этого введем банахово пространство

$$
\mathscr{B}_{p}:=\left\{f(\rho): f(\rho) \rho^{-1} \in L_{p}\left(\omega^{*}\right)\right\}, \quad p>1,
$$

с нормой $\|f\|_{\mathscr{B}_{p}}:=\left\|f(\rho) \rho^{-1}\right\|_{L_{p}\left(\omega^{*}\right)}$. Определим матрицу $D(x, \rho), \rho \in \omega^{*}, x \geqslant 0$, следующим образом: при $\rho \in \omega^{0} D(x, \rho)=\operatorname{diag}\left[D_{k}(x, \rho)\right]_{k=\overline{1, n}}, D_{k}(x, \rho)=\exp \left(-\rho R_{k} x\right), R_{k}=R_{k}\left(S_{j}\right)$; при $\rho \in \omega_{j}^{1} D(x, \rho)=\operatorname{diag}\left[D_{k}(x, \rho)\right]_{k=\overline{1,2 n}}, D_{k}(x, \rho)=\exp \left(-\rho R_{k} x\right)$ для $k \leqslant n$, и $D_{k}(x, \rho)=$ $\exp \left(-\rho R_{k-n} x\right)$ для $k>n ; R_{k}=R_{k}\left(S_{j}\right)$.

TEOPEмA 4. Пусть $M(\rho)$ - матрииа Вейля для nары $L=(\ell, U)$. Пусть пара $\widetilde{L}=(\tilde{\ell}, \widetilde{U})$ выбрана так, чтобы выполнялось (7). Тогда при каждом фиксированном $x \geqslant 0$, основное уравнение (8) имеет единственное решение $\varphi(x, \rho)$ в классе $\varphi(x, \rho) D(x, \rho) \in \mathscr{B}_{p}$ при каждом $p>1 u \sup _{x \geqslant 0}\|\varphi(x, \rho) D(x, \rho)\|_{\mathscr{B}_{p}}<\infty$.

Приведем теперь явные формулы для построения потенциала $Q(x)$. Обозначим

$$
\varepsilon(x)=\frac{1}{2 \pi i} \int_{\omega}\left(\Phi(x, \mu) A_{0}(\mu) \widetilde{\Phi}^{*}(x, \mu) Q_{0}-Q_{0} \Phi(x, \mu) A_{0}(\mu) \widetilde{\Phi}^{*}(x, \mu)\right) d \mu .
$$

Теорема 5. Справедливы соотношения

$$
Q(x)=\widetilde{Q}(x)+\varepsilon(x), \quad h=\tilde{h} .
$$

Таким образом, получаем следующую прочедуру решения обратной задачи. Пусть задана матрица Вейля $M(\rho)$ пары $L=(\ell, U)$.

1) Выберем "модельню" пару $\widetilde{L}=(\tilde{\ell}, \widetilde{U})$ так, чтобы выполнялось $(7)$.

2) Построим матрицы-функции $\tilde{\varphi}(x, \rho), \widetilde{S}(\rho), \tilde{r}(x, \mu, \rho), x \geqslant 0, \mu, \rho \in \omega^{*}$.

3) Решая основное уравнение (8), находим $\varphi(x, \rho)$ при $x \geqslant 0, \rho \in \omega^{*}$, т.е. находим $\Phi(x, \rho)$ при $x \geqslant 0, \rho \in \omega$.

4) Вычисляем матрицу-функцию $\varepsilon(x)$ по формуле (9).

5) Строим пару $L=(\ell, U)$ по формуле (10).

\section{СПИСОК ЦИТИРОВАННОЙ ЛИТЕРАТУРЫ}

1. Левитан Б. М., Саргслн И. С. Операторы Штурма-Лиувилля и Дирака. М.: Наука, 1988. 2. Sakhnovich L. A. Spectral Theory of Canonical Differential Systems. Method of Operator Identities. Operator Theory: Adv. and Appl. V. 107. Basel: Birkhäuser Verlag, 1999. 3. Шaбат А. Б. // Дифференц. уравнения. 1979. Т. 15. №10. С. 1824-1834. 4. Марченко В. А. Операторы Штурма-Лиувилля и их приложения. Киев: Наукова думка, 1977. 5. Левитан Б. М. Обратные задачи Штурма-Лиувилля. М.: Наука, 1984. 6. Freiling G., Yurko V. A. Inverse Sturm-Liouville Problems and Their Applications. New York: NOVA Science Publishers, 2001. 7. Маламуд M. M. // Tp. MMO. 1999. T. 60. C. 199-258. 8. Beals R., Coifman R. R. // Comm. Pure Appl. Math. 1984. V. 37. P. 39-90. 9. Zhou X. // Comm. Pure Appl. Math. 1989. V. 42. P. 895-938. 10. Юрко В. А. // Матем. сб. 1991. Т. 182. №3. С. 431-456. 11. Yurko V. А. Method of Spectral Mappings in the Inverse Problem Theory. Inverse and Ill-posed Problems Series. Utrecht: VSP, 2002. 12. Юрко В. А. Обратные спектральные задачи и их приложения. Саратов: Изд-во Саратовского пединститута, 2001. 13. Yurko V. A. An inverse problem of spectral analysis for systems of differential equations on the half-line. Schriftenreihe des Instituts für Mathematik, SM-DU-556: Universität Duisburg-Essen, 2003. 